\title{
Level-set Method for Robust Analysis of Optical Mapping Recordings of Fibrillation
}

\author{
Daniel R. Gurevich, Conner Herndon, Ilija Uzelac, Flavio H. Fenton, Roman O. Grigoriev \\ Georgia Institute of Technology, Atlanta, Georgia, USA
}

\begin{abstract}
We have developed a level-set based approach that can accurately and reliably extract the temporally and spatially resolved positions of wavefronts, wavebacks, and phase singularities (PSs) from noisy optical mapping data. The algorithm consists of three steps: pointwise phase reconstruction, computation of smooth level sets of the phase field, and identification of phase singularities as the level set intersections. This method was illustrated by performing an analysis of optical mapping voltage data during ventricular fibrillation in a Langendorff-perfused pig heart. Observed behavior of the PSs was in excellent agreement with previous experimental studies: PSs appeared and disappeared in pairs of opposite topological charge, their lifetimes ranged from milliseconds to seconds, and the longest-lived PSs became "pinned" on structural inhomogeneities of the heart tissue.
\end{abstract}

\section{Introduction}

Although re-entry, presently understood as a spiral wave of excitation, has long been implicated as a key ingredient underlying fibrillation, the basic dynamical mechanisms responsible for initiating and maintaining this complex rhythm, which features multiple interacting spiral or scroll waves, are still being debated. The leading theory is that fibrillation is maintained by wave breakup caused by variation of tissue refractoriness [1], which can be static (due to tissue inhomogeneities) or dynamically induced [2]. However, recent numerical evidence suggests that, at least in atrial fibrillation, the dynamics can be described as a dynamical equilibrium between two topologically distinct processes, wave coalescence and wave collapse, but not wave breakup [3].

In order to test these predictions in experiment, a computational algorithm is required that can extract the position of wavefronts, wavebacks, and phase singularities (PSs) from optical mapping data. The phase corresponds to the local state of cardiac cells; this correspondence breaks down at the PSs, where the phase is ill- defined. The traditional definitions of the phase is $\phi(t)=$ $\arctan (\Delta V(t+\tau) / \Delta V(t))$, where $\Delta V=V-V_{0}$, and $V_{0}$ and $\tau$ are a suitably chosen reference voltage and time delay, respectively [4]. The PSs are then defined as points enclosed by small contours along which an approximation of the contour integral $\oint d \mathbf{r} \cdot \nabla \phi$ is roughly equal to $\pm 2 \pi$ [5] [6]. Alternative approaches are to define the PSs as points where the normal velocity of a voltage level set is zero [7] or as local minima of the amplitude of the signal [8]. These approaches are highly unreliable for typically noisy experimental recordings. This paper introduces a new method for detecting PSs, as well as wavefronts and wavebacks, that is substantially more robust and can be used for analysis of both numerical and experimental data.

\section{Methods}

\subsection{Experiment}

All experimental procedures were approved by the Georgia Tech Office of Research Integrity Assurance and T3 Labs. The pig was anesthetized using $5 \mathrm{mg} / \mathrm{kg}$ of xylazine, injected with 300 units $/ \mathrm{kg}$ of heparin, and euthanized using $120 \mathrm{mg} / \mathrm{kg}$ of pentobarbital. The heart was quickly removed via left thoracotomy and the aorta perfused retrogradely with cardioplegic solution. The right ventricle was separated, cannulated, then perfused at $60 \mathrm{mmHg}$ with $37 \pm 1^{\circ} \mathrm{C}$ Tyrode's solution containing (in mM): $\mathrm{NaCl} 124, \mathrm{KCl} 4, \mathrm{NaHCO}_{3} 24, \mathrm{NaH}_{2} \mathrm{PO}_{4} \cdot \mathrm{H}_{2} \mathrm{O} 0.9$, $\mathrm{MgCl} \cdot 6 \mathrm{H}_{2} \mathrm{O} 0.7, \mathrm{CaCl}_{2} \cdot 2 \mathrm{H}_{2} \mathrm{O} 2$, and Glucose 5.5, gassed with $95 \% \mathrm{O}_{2}$ and $5 \% \mathrm{CO}_{2}$. Over the course of $45 \mathrm{~min}$, the heart was stained with $0.5 \mathrm{mg}$ voltage sensitive dye Di-4-ANBDQPQ (JPW6003), and heart motion was suppressed using $1 \mathrm{mg}$ blebbistatin. Tissue was illuminated using three $660 \mathrm{~nm}$ LEDs coupled with band emission filter of 650/10nm (Edmund Optics), and epicardial fluorescence signals were filtered through a 700nm long-pass filter and recorded using a back-illuminated EMCCD camera (Photometrics Evolve 128) at $500 \mathrm{fps}$ and $128 \times 128$ pixels, corresponding to a spatial resolution of approximately $250 \mu \mathrm{m}$ per pixel. Ventricular fibrillation was induced using high frequency pacing from a bipolar electrode current source. 


\subsection{Numerics}

The optical data $I(x, y, t)$ were masked based on the temporal Fourier spectrum of the signal in order to remove the signal from outside the heart. A high-pass filter was applied to remove the slow drift in the data. Then, in order to reduce noise, the signal was smoothed over both space and time using a Gaussian kernel, yielding a field $\bar{I}(x, y, t)$. Each PS is defined by the intersection of two smooth contours. These are defined as different level sets of an appropriately defined phase field $\phi(x, y, t)$ computed independently at each spatial grid point $\left(x_{0}, y_{0}\right)$. Let $t_{1}$ and $t_{2}$ correspond to successive maxima of $\bar{I}\left(x_{0}, y_{0}, t\right)$. On the interval $\left[t_{1}, t_{2}\right)$ the phase is defined as

$$
\phi\left(x_{0}, y_{0}, t\right)=2 \pi \frac{t-t_{1}}{t_{2}-t_{1}} .
$$

This definition is similar to the isochronal map representation [9][10] but further takes into account the period of local dynamics. Close to PSs, the signal is weak compared to noise (see Fig.1). To make the definition of the maxima robust near the PSs, the maximum is defined to occur at time $t_{0}$ only if $\bar{I}\left(x_{0}, y_{0}, t_{0}\right) \geq \bar{I}\left(x_{0}, y_{0}, t\right)$ for all $t \in\left[t_{0}-\Delta t, t_{0}+\Delta t\right]$, where $\Delta t$ is a fixed fraction of the average period of the signal.

Each level set $\phi=$ const terminates at a PS; to create two continuous smooth curves passing through the PSs, the curve $\ell_{1}$ is defined as the union of the level sets with $\phi=0$ and $\phi=\pi$, and the curve $\ell_{2}$ is defined as the union of the level sets $\phi=\pi / 2$ and $\phi=3 \pi / 2$. Since the phase is a (piecewise) linear function of time, the pairs of level sets that are joined together meet at roughly $180^{\circ}$ angles.

These definitions of level sets and PSs use only local information, making them sensitive to noise. We can incorporate global information by modifying the definition of the contours according to the following procedure: First, an unsigned distance function $d_{i}$ is constructed for contour $\ell_{i}$ using a MATLAB implementation of the fast marching method [11] by Gabriel Peyré [12]. The unsigned distance is converted into a signed distance function $d_{i}^{s}$ using the phase:

$$
\begin{aligned}
& d_{1}^{s}(x, y)=d_{1}(x, y) \sin (\phi(x, y)) \\
& d_{2}^{s}(x, y)=d_{2}(x, y) \cos (\phi(x, y))
\end{aligned}
$$

The distance function $d_{i}^{s}$ is then smoothed by applying a 2D spatial low-pass filter, discarding all modes with wavenumbers higher than a set threshold. Last, the zero level set of the smoothed distance function $d_{i}^{*}$ is used to redefine the level set of the phase field, now denoted by $\ell_{i}^{*}$. Each intersection of $\ell_{1}^{*}$ and $\ell_{2}^{*}$ (found using the Fast and Robust Curves Intersections MATLAB function by Douglas Schwarz [13]) defines a PS, the position of which can be determined with subpixel accuracy.
The topological charge (chirality) $q$ of a PS is defined to be +1 if the phase increases going counterclockwise around the singularity and -1 otherwise. Thus, traditionally, $q$ has been computed in terms of the contour integral of the gradient of the phase around the PS [6]. This definition is sensitive to noise and breaks down for closelyseparated pairs of PSs. To address these problems, we instead define chirality using the gradients of the distance functions. Unlike the gradient of the phase, which is illdefined near the PS, the gradient of each distance function is nearly uniform in direction. Applying the right-hand rule to $\nabla d_{1}^{*}$ and $\nabla d_{2}^{*}$, it is easy to see that

$$
q=\operatorname{sign}\left(\hat{\mathbf{z}} \cdot \nabla \mathrm{d}_{1}^{*} \times \nabla \mathrm{d}_{2}^{*}\right),
$$

where the gradients are evaluated at the grid point nearest to the PS.

\section{Results}

This algorithm was tested on optical mapping recordings of ventricular fibrillation in adult pig hearts. Raw (gray) and smoothed (black) voltage data at two different points in the heart are compared in Figure 1. To obtain the smoothed signal, a Gaussian kernel was applied with a standard deviation of two pixels in each spatial direction and three recording frames in time. The data in Fig. 1(a) was captured far from all PSs, whereas Fig. 1(b) shows the signal close to a persistent PS. The voltage in both panels was normalized by the same factor, with the normalized voltage denoted by $v_{m}$. The depressed action potentials in Fig. 1(b) have a much smaller amplitude and thus a significantly lower signal-to-noise ratio. However, in all cases, the smoothed signal has well-defined peaks, which is important in order to ensure that the algorithm yields accurate level sets.

Results from a particular 10-second long recording are shown in Figure 2. The panels show equally spaced snapshots of the raw voltage data over an interval that roughly corresponds to one period of rotation of the spiral waves. Overlaid on the voltage field are the level sets $\ell_{1}^{*}$ (white) and $\ell_{2}^{*}$ (black) and the PSs, which correspond to the intersections of these level sets.

The figure clearly illustrates different types of behavior exhibited by the PSs. Some of the PSs, such as the PS marked by an arrow in Fig. 2(a), are long-lived and remain stationary for up to seconds, becoming a source of a persistent spiral wave (mother rotor). Other PSs, such as the ones marked by arrows in Fig. 2(c), are short-lived, appearing and disappearing in pairs with opposite chirality within tens of milliseconds. Occasionally, single PSs, e.g., PS marked by an arrow in Fig. 2(b), are destroyed when they collide with the boundary of the heart.

Long-lived PSs tend to be attracted to the same tissue regions, suggesting the presence of structural hetero- 


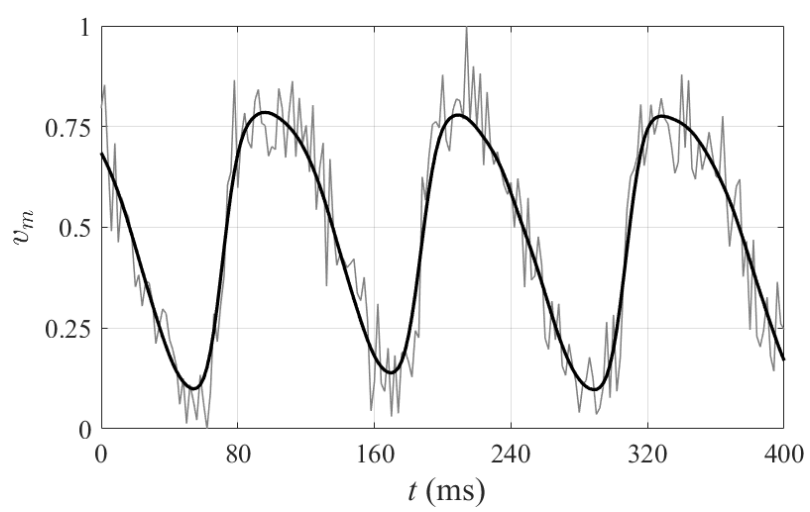

(a)

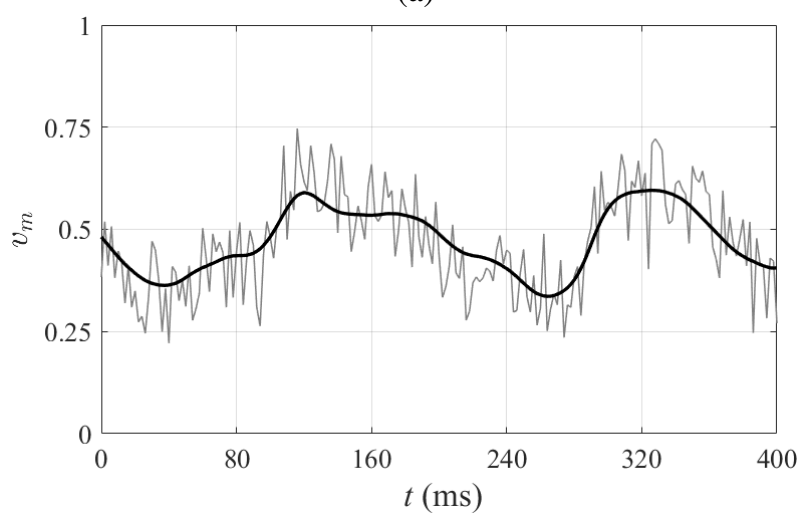

(b)

Figure 1. Comparison of raw (gray) and smoothed (black) voltage data over a $400 \mathrm{~ms}$ period, (a) at a point far from all PSs and (b) at a point close to a persistent PS. Both signals have been normalized by the same factor.

geneities. To quantify this we computed the probability density function (PDF)

$$
P(x, y)=N(x, y) / \sum_{x^{\prime}, y^{\prime}} N\left(x^{\prime}, y^{\prime}\right),
$$

where $N\left(x^{\prime}, y^{\prime}\right)$ is the total number of frames containing a phase singularity in the cell with coordinates $\left(x^{\prime}, y^{\prime}\right)$. The PDF $P(x, y)$ and the trajectories of the four longest-lived PSs (with lifetimes ranging from $1.6 \mathrm{~s}$ to $4.4 \mathrm{~s}$, i.e., between roughly 15 and 40 revolutions) are shown in Fig. 3. These PSs tend to stay "pinned" for extended periods of time in the immediate neighborhood of three sharp maxima. Such behavior has been frequently observed in other experimental studies [14]. In addition, we find thin linear ridges with high values of $P(x, y)$; shorter-lived PSs (not shown) tend to move along these ridges without getting "pinned."

The level sets $\phi=0, \phi=\pi / 2, \phi=\pi$, and $\phi=3 \pi / 2$ by themselves also provide a useful visualization of the dynamics. Figure 4 shows the correspondence between the normalized smoothed voltage signal $v_{m}$ and the phase at

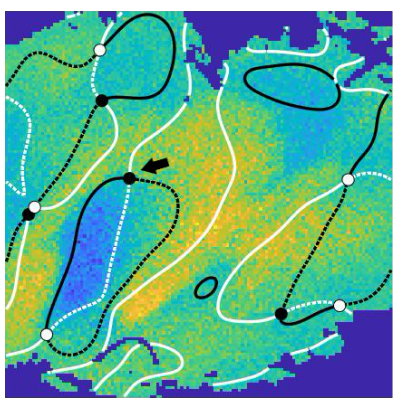

(a)

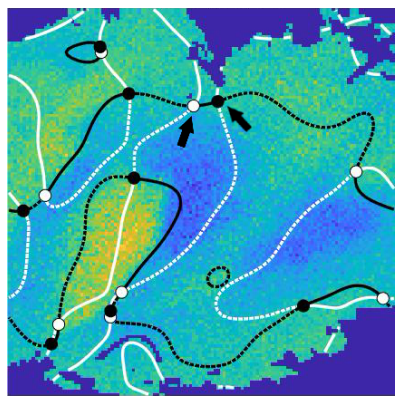

(c)

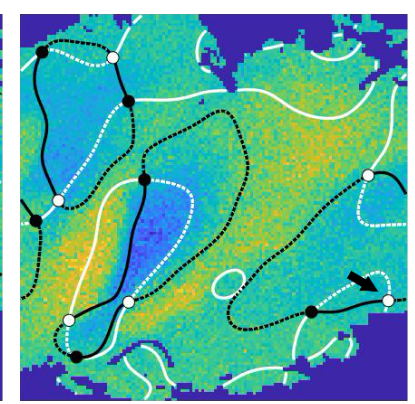

(b)

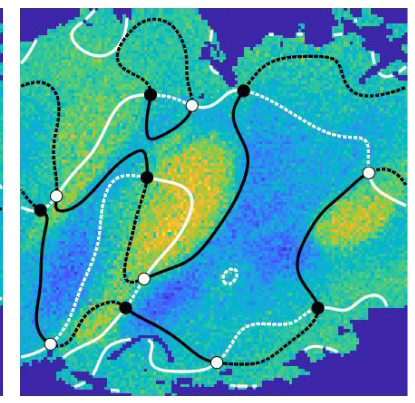

(d)
Figure 2. Four snapshots of raw optical mapping voltage data and the corresponding level sets and PSs. Level sets with phases $0, \pi / 2, \pi$, and $3 \pi / 2$ are plotted as solid white, dashed black, dashed white, and solid black lines, respectively, and PSs with chirality +1 and -1 , as black and white circles, respectively. The masked areas are shown in dark blue. The snapshots were taken $28 \mathrm{~ms}$ apart.

the center of the imaged region over the course of one typical period. By definition, the $\phi=0$ level set corresponds to the leading edge of the refractory region and $\phi=\pi$ approximately to its trailing edge [3]. The $\phi=3 \pi / 2$ and $\phi=\pi / 2$ level sets correspond approximately to the wavefront and the waveback. Even more accurate approximations could be easily constructed by choosing level sets that correspond to other values of $\phi$ for specific dynamical regimes or tissue types. It is important to note that the choice of phase level sets does not affect the position of the phase singularities. Equally importantly, choosing wavefronts to correspond to a level set $\phi=$ const allows a formal and rigorous definition of wavelets [15], each of which terminates at two PSs of opposite chirality.

\section{Conclusions}

We have demonstrated that the level sets of the phase field allow a convenient and robust definition of the key topological features of excitation patterns characterizing complex cardiac arrhythmias. Since suitably defined level sets can incorporate global information about the phase field, their shape and their intersections, which define 


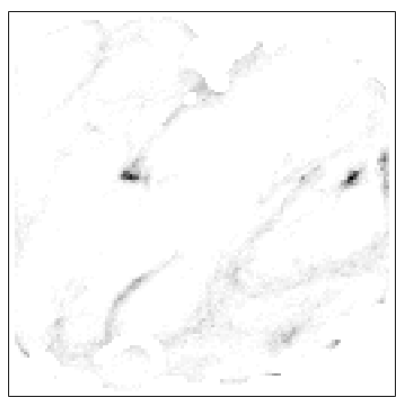

(a)

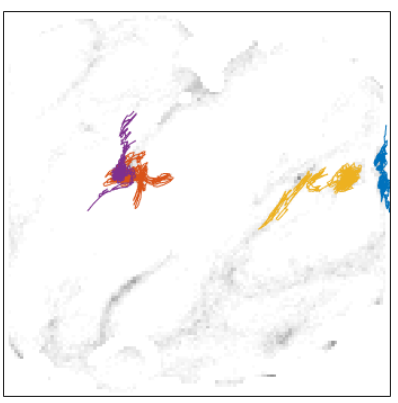

(b)
Figure 3. Spatial distribution of PSs. (a) PDF $P(x, y)$ describing likelihood of finding a PS at each location, with black (white) corresponding to the highest (lowest) probability. (b) Trajectories of the four longest-lived PSs (distinguished by color) overlaid on $P(x, y)$.

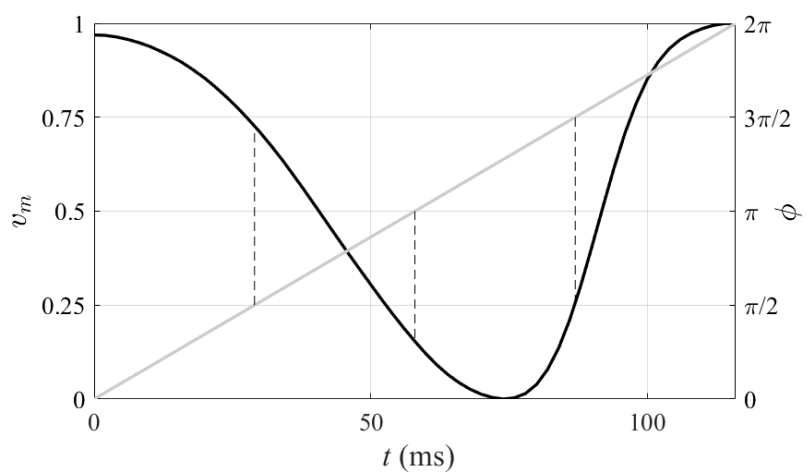

Figure 4. Normalized smoothed voltage (black) and phase (gray) over one typical period. Dashed lines indicate where $\phi=\pi / 2, \pi$, and $3 \pi / 2$.

PSs, are extremely robust with respect to noise. We have demonstrated this using an optical mapping recording of fibrillation in a pig heart. The analysis of the dynamics reflects both the presence of long-lived mother rotors with nearly stationary cores and the spontaneous births and collapses of pairs of PSs at the core of much more mobile and short-lived spiral waves. These results illustrate the promise of our approach in analyzing dynamics and patterns in a variety of applications.

\section{Acknowledgements}

This material is based upon work supported by the National Science Foundation under Grants No. 1341190, 1028133, 1341128, 1347015 and the American Heart Association under Grant No. 15POST25700285. DG was supported by the Petit Undergraduate Research Scholarship.

\section{References}

[1] Moe GK. On the multiple wavelet hypothesis of atrial fibrillation. Arch Int Pharmacodyn Ther 1962;140:183-188.

[2] Cherry EM, Fenton FH. Visualization of spiral and scroll waves in simulated and experimental cardiac tissue. New $\mathbf{J}$ Phys 2008;10(12):125016.

[3] Marcotte CD, Grigoriev RO. Dynamical mechanism of atrial fibrillation: a topological approach. Chaos 2017; 27(9):093936.

[4] Gray RA, Pertsov AM, Jalife J. Spatial and temporal organization during cardiac fibrillation. Nature 1998; 392(6671):75-78.

[5] Iyer AN, Gray RA. An experimentalist's approach to accurate localization of phase singularities during reentry. Ann Biomed Eng 2001;29(1):47-59.

[6] Bray MA, Lin SF, Aliev RR, Roth BJ, Wikswo JP. Experimental and theoretical analysis of phase singularity dynamics in cardiac tissue. J Cardiovasc Electrophys 2001; 12(6):716-722.

[7] Fenton F, Karma A. Vortex dynamics in three-dimensional continuous myocardium with fiber rotation: Filament instability and fibrillation. Chaos 1998;8(1):20-47.

[8] Byrne G, Marcotte CD, Grigoriev RO. Exact coherent structures and chaotic dynamics in a model of cardiac tissue. Chaos 2015;25(3):033108.

[9] Chen PS, Garfinkel A, Weiss JN, Karagueuzian HS. Computerized mapping of fibrillation in normal ventricular myocardium. Chaos 1998;8(1):127-136.

[10] Lee MH, Qu Z, Fishbein GA, Lamp ST, Chang EH, Ohara T, Voroshilovsky O, Kil JR, Hamzei AR, Wang NC, et al. Patterns of wave break during ventricular fibrillation in isolated swine right ventricle. Am J Physiol 2001; 281(1):H253-H265.

[11] Sethian JA. A fast marching level set method for monotonically advancing fronts. Proceedings of the National Academy of Sciences 1996;93(4):1591-1595.

[12] Peyré G. Fast marching in 2D. http: //www. numerical-tours.com/matlab/ fastmarching_1_2d/.

[13] Schwarz D. Fast and robust curve intersections. http: //www.mathworks.com/matlabcentral/ fileexchange/11837-fast-and-robustcurve-intersections.

[14] Pertsov AM, Davidenko JM, Salomonsz R, Baxter WT, Jalife J. Spiral waves of excitation underlie reentrant activity in isolated cardiac muscle. Circ Res 1993;72(3):631-650.

[15] Chen J, Mandapati R, Berenfeld O, Skanes AC, Gray RA, Jalife J. Dynamics of wavelets and their role in atrial fibrillation in the isolated sheep heart. Cardiovasc Res 2000; 48(2):220-232.

Address for correspondence:

Daniel Gurevich

School of Physics, Georgia Institute of Technology, Atlanta, USA dgurevich6@gatech.edu 